\title{
The golden generation in Portuguese nephrology: fact or fiction?
}

\author{
Marco Mendes (iD \\ Nephrology Department, Hospital Curry Cabral; Lisbon, Portugal
}

The score of 19 has become trivialized during Nephrology residence evaluation or as a final exam grade for some time. However, in recent years we have witnessed a dizzying rise in these grades all over the country, to the point that candidates to Nephrology final exam are now ranked at the scale of hundredths with scores of 19.92, 19.90, 19.8 and so on.

Is this a reflection of reality, with the improvement of internship programs and a generation of future Nobel prizewinners, or are we losing criteria in the evaluation of the resident?

The fact that gaining a position as consultant has become dependent exclusively on the final exam score has triggered this grade inflation, as juries tend to assume that these inflated grades promote equity between candidates. We have reached a point when a candidate with a final score below 19.5 is at the tail of the class limited options.

Residence grades have also become decisive due to their $40 \%$ contribution to the final grade of the curricular exam, which can create an insurmountable gap between candidates from the 1st day of the exam. Hence, there is an identical inflation of these scores by the respective departments so as not to harm the house's residents.

Since the score is so decisive, no jury wants to harm the group they are examining so, understandably, tends to become more generous. Each jury is different, unaware of what is happening in the other juries, and like so, do not articulate criteria between then. This way, there is a risk that the residents will be ranked by the place of the examination and not by the examination itself. A brilliant resident / exam at site $X$ may fall below a mediocre candidate at site Y. For years, the College of Nephrology determined two common elements to all exams that at least served as a point of balance between them.

The way we are evaluating young specialists has become increasingly anarchic and liable to be unfair, with the risk that the final exam won't reflect the merits. There is an urgent need to reform this evaluation.

Similar to what happens in other specialties, the different internships during residence could be evaluated in written exams equal to every candidates. The final specialty exam could maintain an evaluation of the curriculum and a practical exam with the current characteristics, as it is essential to assess the interaction of the intern with his peers and see his approach to the patient. However, there would be an independent jury for the two tests so that the performance in one would not influence the jury in the second. The same jury would evaluate all candidates in each category so they could be fairly scored. The theorical exam would be of multiple choice, with a similar model to the European exam. At present, theoretical knowledge shown is often camouflaged by the expression capacity of the candidates, already assessed in the discussion of the curriculum and in the practical test. In addition, a multiple choice exam would ensure that all interns answer on the same topics and exclude bias in question selection.

These suggestions are just a few of the many changes that can be instituted. But something must be done as soon as possible to stop this vertiginous and absurd rise in the scores that, instead of making us proud, should make us apprehensive. In addition, it is a score that is on the curriculum of each consultant and effective for any competition that he wants to apply in the future. A nephrologist, with a meritorious 19 a few years ago, is today completely cut off from any open competition. This is not acceptable.

It is up to the entire nephrological community, including interns and specialists, together with the College to find and implement changes so that the fellowship grades and final assessments become fairer, uniform and credible. We must not vulgarize excellence but must assure a just and equal evaluation of our young nephrologists, which is essential to define their future.

Disclosure of potential conflicts of interest: none declared.

ORCID

Marco Mendes (iD) 0000-0002-5109-0697

\author{
Correspondence to: \\ Marco Mendes, MD \\ Nephrology Department, Hospital Curry Cabral \\ Centro Hospitalar Universitário de Lisboa Central \\ E-mail: marcomendes82@gmail.com
}

